\title{
A theoretical derivation of the transients related to partial discharges in ellipsoidal voids
}

Crichton, George C; Karlsson, A.; Pedersen, Aage

Published in:

I E E E International Symposium on Electrical Insulation. Conference Record

Link to article, DOI:

10.1109/ELINSL.1988.13913

Publication date:

1988

Document Version

Publisher's PDF, also known as Version of record

Link back to DTU Orbit

Citation (APA):

Crichton, G. C., Karlsson, A., \& Pedersen, A. (1988). A theoretical derivation of the transients related to partial discharges in ellipsoidal voids. I E E E International Symposium on Electrical Insulation. Conference Record, 238-241. https://doi.org/10.1109/ELINSL.1988.13913

\section{General rights}

Copyright and moral rights for the publications made accessible in the public portal are retained by the authors and/or other copyright owners and it is a condition of accessing publications that users recognise and abide by the legal requirements associated with these rights.

- Users may download and print one copy of any publication from the public portal for the purpose of private study or research.

- You may not further distribute the material or use it for any profit-making activity or commercial gain

- You may freely distribute the URL identifying the publication in the public portal 
Conference Record of the 1988 IEEE International Symposium on Electrical Insulation, Boston, MA, June 5-8, 1988 A THEORETICAL DERIVATION OF THE TRANSIENTS RELATED TO PARTIAL DISCHARGES IN ELLIPSOIDAL VOIDS

\author{
G.C. Crichton, P.W. Karlsson and A. Pedersen \\ Physics Laboratory II, Building 309B, \\ The Technical University of Denmark, \\ DK-2800 Lyngby, Denmark.
}

ABSTRACT. Transients associated with partial discharges in ellipsoidal and spheroidal voids are derived in terms of the concept of induced charges on the e1ectrode. The relationship between the induced charge and the properties which are usually measured are discussed. The method is illustrated by applying it to a spheroidal void in a simple disk-type GIS spacer.

\section{INTRODUCTION}

The transients which are manifest at the electrodes of a system with partial discharges are related to the charges which, in view of Faraday's ice pail experiment, are induced on the electrodes. The sources of the induced charges are the charges which are distributed within the voids as a result of partial discharges.

The induced charge can be expressed as the difference between the charge on the electrode when discharges have occurred and the charge which would have been on the electrode had the system been discharge free $|1|$.

A more straightforward approach is possible through an application of the principle of superposition $|2,3|$. This can be done in two different ways depending on whether emphasis is placed on the P-field |2| or on the $D$-field $|3|$ in the dielectric. In practice the application of the latter is more convenient and this approach will therefore be employed in the present paper.

A discharge in a void results in a deployment of charges on the surface $S$ of the void. The surface charge density $\sigma$ will attain such values that the field within the void is reduced until the discharge is quenched. The induced charge related to the charge distribution on $S$ is given by $|3|$

$$
q=-\int_{S} \sigma \lambda \mathrm{d} S
$$

in which $\lambda$ is a dimensionless scalar function which depends on the position of $\mathrm{d} S$ only. The function $\lambda$ is given by Laplace's equation

$$
\vec{\nabla} \cdot(\varepsilon \vec{\nabla} \lambda)=0
$$

where $\varepsilon$ is the permittivity. The boundary conditions are: $\lambda=1$ at the electrode on which $q$ is distributed and $\lambda=0$ at all other electrodes. In addition the following condition must be fulfilled at all dielectric interfaces

$$
\varepsilon_{+}(\partial \lambda / \partial n)_{+}=\varepsilon_{-}(\partial \lambda / \partial n)_{-}
$$

where $\lambda$ is differentiated in the direction normal to the interface and + and - refer to the two sides of the interface. Since Eq.(2) is Laplace's equation any standard method for calculation of space charge free electrostatic fields can be used to calculate $\lambda$.

The charge pattern which is deposited on $S$ can norma11y be considered as an electric dipole since the nett charge within the void remains zero. The dipole moment $\vec{\mu}$ is given by

$$
\vec{\mu}=\int_{S} \sigma \overrightarrow{r^{2}} \mathrm{~d} S
$$

where $\vec{r}$ is a vector which locates the position of $\mathrm{d} S$. The induced charge from this dipole becomes $|3|$

$$
q=-\vec{\mu} \cdot \vec{\nabla} \lambda
$$

Since voids in epoxy spacers are usually close to spherical in shape it is of interest to consider transients caused by discharges in an ellipsoidal or spheroidal void. Formulae for the dipole moment of relevant charge distributions on ellipsoids are given. The effects of size, shape and location of the void are discussed with special reference to spheroidal voids.

\section{TRANSIENTS RELATED TO INDUCED CHARGES}

A1though the observable transients are inherently related to the induced charge the properties which primarily are measured are transients in the applied voltage and current pulses in the lead to the electrode. The connection between these properties and the induced charge can be found in the following way. Just prior to the first discharge in the void the potential of the electrode is $U$ and the charge is $Q$. We compare this with the situation immediately after the discharge is quenched when the potential has dropped to $U-\Delta U$ and the charge has become $Q+\Delta Q$, where $\Delta Q$ is the charge transferred to the electrode from the external circuit. Green's reciprocation theorem then yields

$$
Q(U-\Delta U)=(Q+\Delta Q) U+\int_{S} \sigma V \mathrm{~d} S
$$

where $V$ is the scalar potential at the surface element $\mathrm{d} S$ for the discharge free situation. Since $V=\lambda U$, in consequence of equations (2) and (3), and $Q=C V$, where $C$ is the capacitance of the system, we obtain

$$
-\int_{S} \sigma \lambda \mathrm{d} S=C \Delta U+\Delta Q
$$

or

$$
q=C \Delta U+\Delta Q \text {. }
$$

If the impedance of the circuit is large for the current which is associated with the discharge then $C \Delta U$ can be much larger than $\Delta Q$. The induced charge is then approximate $1 \mathrm{y}$

$$
q=C \Delta U \text {. }
$$

It should be emphasized that the capacitance of the system is not affected by partial discharges 2,3 and that, consequently, the transients cannot be related to a change in the capacitance. The proper concept of capacitance 4 implies that the field between the electrodes is Laplacian, and the field is not Laplacian if space charges are present. However, utilizing the principle of superposition the actual field can be considered to be the sum of a space charge Poisson field and the original space charge free Laplacian field. It is the latter which represents the proper capacitance of the system. 


\section{ELLIPSOIDAL VOIDS}

We consider an ellipsoidal void the dimensions of which are so small that the internal field may be considered to be nearly uniform. Since any direction of the field within the ellipsoid can be resolved into three fields parallel to the three major axes, it is sufficient to consider the case for which the field is parallel to one of the axes.

A partial discharge can develop when the field within the ellipsoid reaches the inception value $E_{i}$. A discharge will result in a deposition of charges on the surface of the ellipsoid and the field is reduced. The discharge is quenched when the field is reduced to the limiting value $E_{\ell}$, i.e. the field below which ionization growth is impossible. To simplify the analysis we assume that the field within the ellipsoid remains uniform, i.e. the entire volume of the ellipsoid is involved in the discharge. Even if this assumption may not be fulfilled in practice the general conclusions which can be drawn from the analysis will remain valid. The electrostatics of ellipsoids is discussed in several textbooks, e.g. $|5|$. From the field expressions there given it can be proved that the dipole moment of the charge distribution left on the surface of the ellipsoid by the discharge may be written in the form

$$
\vec{\mu}=\frac{8 \pi \varepsilon}{3 A}\left[\vec{E}_{0}-\left(1+\frac{a b c A\left(\varepsilon_{0}-\varepsilon\right)}{2 \varepsilon}\right) \vec{E}_{\ell}\right]
$$

where $a, b, c$ are the major semi-axes of the ellipsoid, $E_{0}$ is the ambient field when the internal field is equal to the inception field $E_{i}$; i.e. $E$ is the field at the location of the ellipsoidal void for the idealized system with no voids present. $E_{0}, E_{i}$ and $E_{\ell}$ are a11 parallel to the a-axis. The parameter $A$ is given by the integral

$$
A=\int_{0}^{\infty} \frac{\mathrm{d} s}{\left(a^{2}+a\right)^{3 / 2} \sqrt{b^{2}+s} \sqrt{c^{2}+s}}
$$

where $s$ is a dumy variable. The relationship between $E_{0}$ and the inception field $E_{i}$ is given by

$$
\vec{E}_{0}=\left(1+\frac{a b e A\left(\varepsilon_{0}-\varepsilon\right)}{2 \varepsilon}\right) \vec{E}_{i},
$$

$\varepsilon$ is the permittivity of the ambient dielectric and $\varepsilon$ is the permittivity of the gas within the ellipsoid; normally $\varepsilon_{0}$ can be assumed to be the permittivity of free space.

If we introduce dimensionless parameters

$$
K=\frac{2}{a b c A}
$$

and

$$
k=\left(1+\frac{a b c A\left(\varepsilon_{0}-\varepsilon\right)}{2 \varepsilon}\right)^{-1}
$$

we may rewrite the dipole moment as

$$
\vec{\mu}=(K / k) \Omega \varepsilon\left(\vec{E}_{i}-\vec{E}_{\ell}\right)
$$

where $\Omega$ equals the volume $(4 \pi / 3) a b e$ of the ellipsoid. The parameters $K$ and $k$ are related by

$$
k=\frac{K \varepsilon_{r}}{1+(K-1) \varepsilon_{r}}
$$

where $\varepsilon_{r}=\varepsilon / \varepsilon_{0}$ is the relative permittivity.

The induced charge is found by combining equations (5) and (15):

$$
q=-(K / k) \Omega \varepsilon\left(\vec{E}_{i}-\vec{E}_{\ell}\right) \cdot \vec{\nabla} \lambda .
$$

Since $\lambda$ is proportional to the scalar potential for the space charge free field we have, with reference to equations (12) and (14), that

$$
\vec{\nabla} \lambda=k \vec{\nabla} \lambda_{0}
$$

where $\lambda_{0}$ is the solution to Laplace's equation, i.e. equation (2), at the location of the ellipsoid for an idealized system with no voids present. The induced charge thus becomes

$$
q=-K \Omega \varepsilon\left(\vec{E}_{i}-\overrightarrow{E_{\ell}}\right) \cdot \vec{\nabla} \lambda_{o} .
$$

\section{SPHEROIDAL VOIDS}

When $b=c$, the ellipsoid becomes a spheroid, and $A$ and $K$ are then expressible in terms of $1 n$, arctan and simpler functions. The dimensionless parameter $K$ for spheroids is given as a function of the axis ratio $a / b$ in Fig. 1 .

The inception field depends on the pressure $p$ of the gas which is contained in the void and on the critical avalanche length. Since the field is parallel to the $\alpha$-axis the minimum value of $E_{i}$ will, for an electron attaching gas, be given by the Paschen-curve

$$
\frac{E_{i}}{p}=\left(1+\frac{M}{2 a p}\right) \frac{E_{l}}{p}
$$

where $M$ is the figure of merit for the gas $|6|$. For $\mathrm{SF}_{6} M=0.040$ bar $\mathrm{mm}$ and $E_{2} / p=8.86 \mathrm{kV} /(\mathrm{mm}$ bar $)$. Similarly we have for non-attaching gases

$$
\frac{E_{i}}{p}=\left(1+\frac{B}{\sqrt{2 a p}}\right) \frac{E_{\ell}}{p}
$$

where $B$ is a constant which is characteristic for the gas $|7|$. For air $B=0.86 \sqrt{\mathrm{mm} \mathrm{bar}}$ and $E_{\ell} / p=2.42$

$\mathrm{kV} /(\mathrm{mm}$ bar). From equations (20) and (21) it is seen that

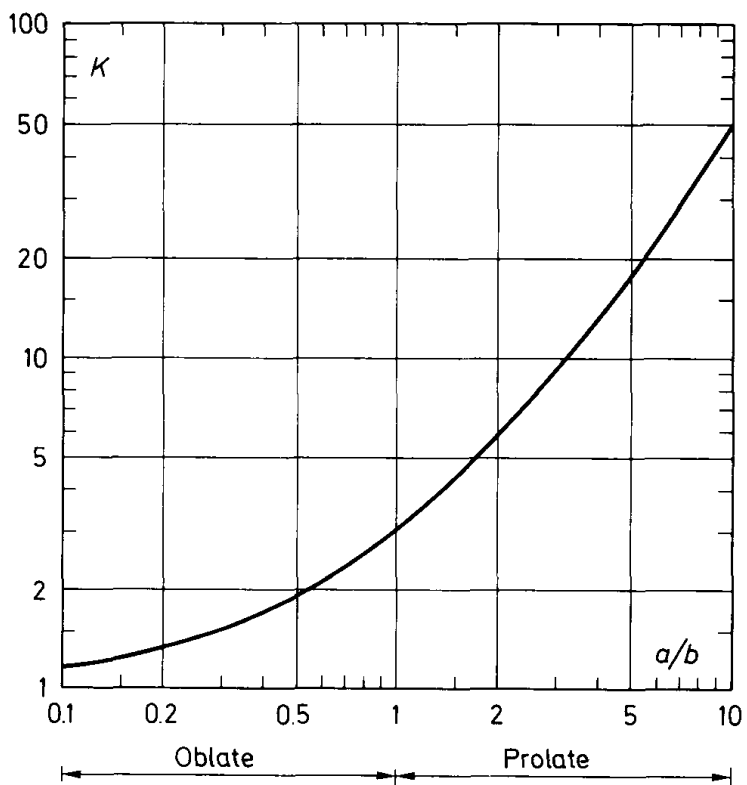

Figure l. The parameter $K$ for spheroids. The applied field is parallel to the a-axis. 


$$
E_{i}-E_{\ell}=M E_{\ell} /(2 a p)
$$

for an electron attaching gas, and that

$$
E_{i}-E_{\ell}=B E_{\ell} / \sqrt{2 a p}
$$

for a non-attaching gas. It should be noted that for an attaching gas $E_{i}-E_{\ell}$ is independent of the pressure, since $E_{\ell}$ is proportional to the pressure.

For a fixed location of the void in the system $\vec{\nabla} \lambda_{0}$ will be constant. Keeping also the volume of the spherofd constant we will study the variation of the induced charge with the ratio $a / b$ between the axes. The induced charge can then be written in the form

$$
q=k_{\mathbf{s}} q_{1}
$$

where $q_{1}$ is the induced charge for a spherical void and $k_{s}$ is a dimensionless shape factor which depends on $a / b$. Insertion of an expression for the semiaxis $a$ in terms of $\Omega$ and $a / b$ in equations (19), (22) and (23) shows that

$$
k_{\mathrm{s}}=\frac{K}{3}\left(\frac{a}{b}\right)^{-2 / 3}
$$

for an electron attaching gas, and

$$
k_{s}=\frac{K}{3}\left(\frac{a}{b}\right)^{-1 / 3}
$$

for a non-attaching gas. The spheroid shape factors are thus independent of a11 the other properties of the gases which may be confined within the spheroid. The shape factors as a function of $a / b$ are shown in Fig. 2.

\section{VOID IN A DISK-TYPE SPACER}

Under consideration is a coaxial electrode system with a simple disk-type spacer made of a dielectric

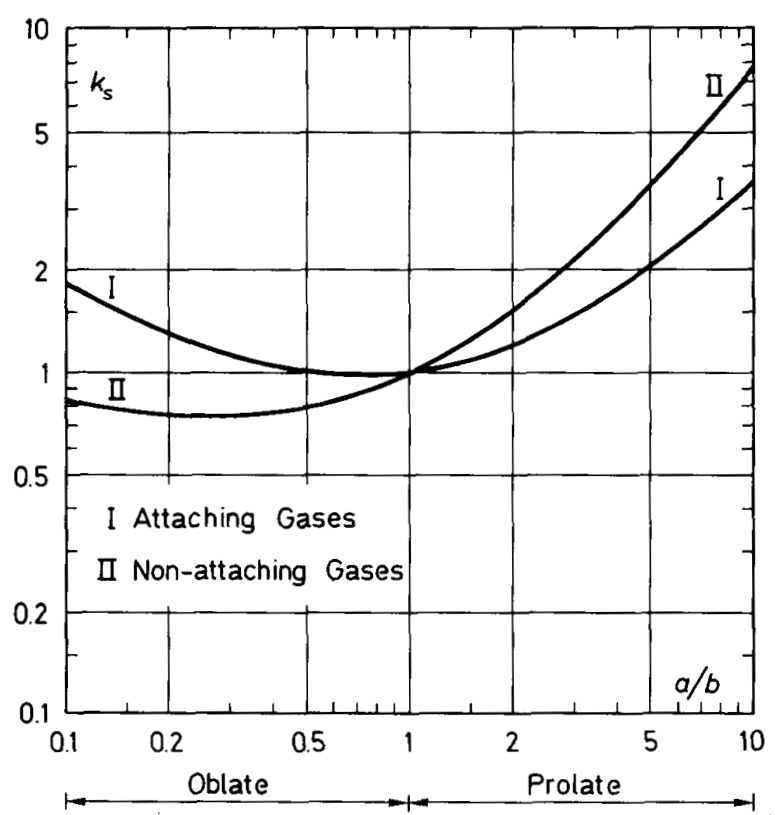

Figure 2. The shape factor $k$ for spheroidal voids of constant volume. with $\varepsilon_{r}=4$. The radius of the inner electrode is $r_{1}=$ $70 \mathrm{~mm}$, and the inner radius of the outer electrode is $r_{2}=190 \mathrm{~mm}$. In the spacer is a spheroidal void of volume $\Omega=1 \mathrm{~mm}^{3}$. The center of the spheroid is located at $r=100 \mathrm{~mm}$ from the axis of the coaxial system. In the void is either $S_{6}$ or air both at a pressure of 1 bar. For this simple geometry

$$
\vec{\nabla} \lambda_{\mathrm{o}}=-\frac{\vec{e}_{\mathrm{r}}}{r \ln \left(r_{2} / r_{1}\right)}
$$

where $\vec{e}_{r}$ is a unit vector perpendicular to the axis of the coaxial system and directed away from the inner electrode.

Insertion of these data in equations (19), (22)(27) gives the induced charge as a function of $a / b$. The results are shown in Fig. 3. The corresponding discharge inception voltages can be calculated from data obtained from equations (20) and (21) and they are shown in Fig.4. It is seen that although the inception voltage is larger for $S_{6}$ than for air, the induced charge is much lower for the $\mathrm{SF}_{6}$-filled void than for the void filled with air. The reason is that $E_{i}-E_{\ell}$ is much smaller for $\mathrm{SF}_{6}$ than for air when $p=1$ bar.

\section{CONCLUSIONS}

The classical philosophy concerning the transients which are related to partial discharges is based on the assumption that the capacitance of the system is

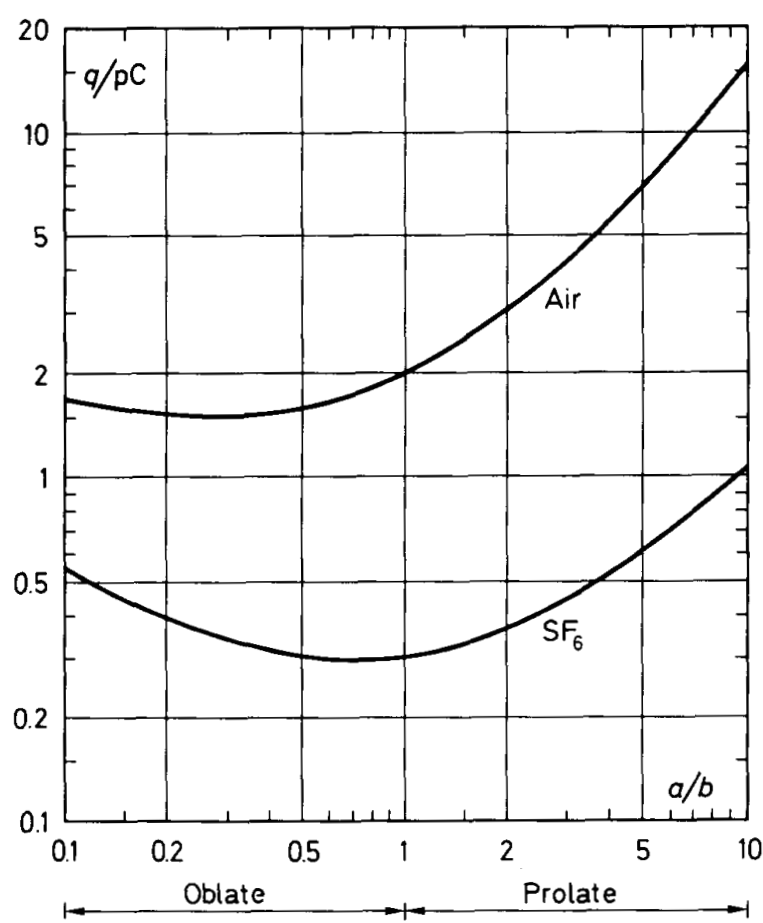

Figure 3. Induced charge $q$ for a spheroidal void in a disk-type spacer. Location: $r=100 \mathrm{~mm}$ from the system axis. Volume: $1 \mathrm{~mm}^{3}$. Electrode dimensions: $r_{1}=70 \mathrm{~mm}, r_{2}=$ $190 \mathrm{~mm}$. Relative permittivity of dielectric: $\varepsilon_{r}=4$. Pressure within the void: 1 bar. 


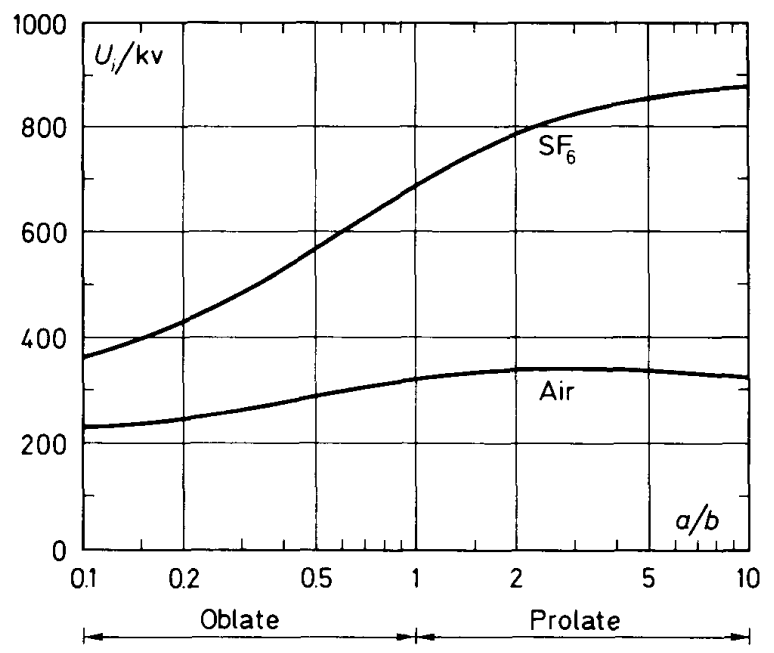

Figure 4. Corona inception voltages $U_{i}$ for the voids referred to in Fig. 3 .

affected by the space charges which are formed by a partial discharge. This is, however, at variance with the proper concept of capacitance. The key to the electrodynamics of partial discharges is the concept of induced charges. Based on this concept analytical expressions are derived for the induced charges related to partial discharges in an ellipsoidal void.

The application of this concept to actual insulating systems is illustrated by considering a spheroidal void in a simple disk-type spacer.

From the formulae obtained conclusions can be drawn about the effects of the gas within the void as well as the effects of size, shape and location of the void.

For the specific case considered the non-attaching gas generates an induced charge which is approximately an order of magnitude larger than that generated by the attaching gas. Inception voltages, however, are higher in the latter case by a factor of about 1.5 to 3.

The yariation in induced charge with location is given by $\vec{\nabla} \lambda$ which depends on the location in the same way as the field strength in the Laplacian field for the idealized system where voids are absent. For a simple disk-type spacer the induced charge is therefore proportional to the inverse of the distance from the axis of the electrode system to the center of the void.

\section{ACKNOWLEDGEMENT}

The authors wish to thank the Electric Power Research Institute for supporting this work under contract RP 2669-I.

\section{REFERENCES}

1 1 H. Repp, K.W. Nissen and P. Röhl, "Partial Discharges in Voids: Inception Conditions and Detection Limits", Siemens Forsch.- u. Entwickl.-Ber., Vol.12, pp. 101-106, 1983.

$|2|$ A. Pedersen, "Current Pulses Generated by Discharges in Voids in Solid Dielectrics. A Field Theoretical Approach", Conference Record of the 1986 IEEE International Symposium on Electrical
Insulation, pp. 112-114, Publication 86CH2196-4DEI, IEEE, 1986.

|3| A. Pedersen, "Partial Discharges in Voids in Solid Dielectrics. An Alternative Approach", Annual Report 1987 IEEE Conference on Electrical Insulation and Dielectric Phenomena, pp. 58-64, Publication 87CH2462-0, IEEE, 1987.

14 J. Fischer, Elektrodynamik. Berlin: Springer-Verlag, 1976

5| J.A. Stratton, Electromagnetic Theory. New York and London: McGraw-Hil1, 1941.

|6| A. Pedersen, I.W. McAllister, G.C. Crichton and S. Vibholm, "Formulation of the Streamer Breakdown Criteria and its Application to Strongly Electronegative Gases and Gas Mixtures", Archiv für Elektrotechnik, Vol.67, pp. 395-402, 1984.

17| I.W. McAllister and A. Pedersen, "Corona-Onset Field-Strength Calculations and the Equivalent Radius Concept", Archiv für Elektrotechnik, Vol. 64 , pp. 43-48, 1981 . 